\title{
Oral manifestation of lepromatous leprosy: diagnosis and management
}

\author{
J. P. S. Servato - L. F. Barbosa De Paulo • \\ P. R. De Faria $\cdot$ S. V. Cardoso $\cdot$ A. M. Loyola
}

Received: 23 March 2014/ Accepted: 10 May 2014/Published online: 22 May 2014

(C) Springer-Verlag Berlin Heidelberg 2014

A 40-year-old man complained about having asymptomatic non-healing oral ulcers for the past 6 months. His familial and medical histories were unremarkable; serological tests were negative for HIV. Physical examination revealed soft, pink granulous lesions involving the upper lip (Fig. 1a) and anterior edentulous alveolar ridge (Fig. 1b). There were also shiny patches on the skin of the back, chest, abdomen, and upper and lower limbs (Fig. 1c). A provisional clinical diagnosis of granulomatous disease disease (such as paracoccidioidomicosis, syphilis, leishmaniasis, tuberculosis and histoplasmosis) was raised, and an incisional biopsy of the oral lesion was performed. Histopathologic sections stained with hematoxylin and eosin (H\&E) revealed epithelial hyperplasia and intense poorly defined granulomatous chronic inflammation with rare giant cells in the lamina propria, without signs of necrosis (Fig. 2a, b). Ziehl-Neelsen staining $(\times 400)$ showed numerous acid-fast bacilli arranged in globi (Fig. 2c). The qPCR test confirms the presence of the Mycobacterium leprae DNA in this sample.

In light of these symptoms, the patient has been diagnosed with multibaccilar/lepromatous leprosy. The patient has been treated with dapson, rifampicin, and clofazimine for 12 months; the oral and cutaneous lesions have been in

J. P. S. Servato · L. F. Barbosa De Paulo .

S. V. Cardoso · A. M. Loyola ( $\square)$

Oral Pathology Area, School of Dentistry,

Faculdade de Odontologia, Federal University of Uberlândia,

Av. Pará, 1720-Campus Umuarama CEP,

38400-902 Uberlândia, MG, Brazil

e-mail: loyolaam@yahoo.com.br

P. R. De Faria

Department of Morphology, Biomedical Science Institute,

Federal University of Uberlândia, Uberlândia, MG, Brazil persistent remission after treatment. Oral leprosy is very uncommon and is largely unknown to most clinicians. As herein reported, clinicians must include it in differential diagnosis of oral granulomatous lesions, and special care must been taken, since these oral lesions could be the first manifestation of systemic disease involvement [1-4]. Incisional biopsy of oral lesions associated with special staining and qPCR investigation were useful and necessary to confirm leprosy diagnosis, prognosis, and treatment [1].

Acknowledgments The authors thank Fundação de Amparo a Pesquisa do Estado de Minas Gerais-FAPEMIG and Conselho Nacional de Desenvolvimento Científico e Tecnológico- CNPq.

Conflict of interest We affirm that we have no financial affiliation (e.g., employment, consultancies, stock ownership, honoraria, paid expert testimony, patent applications/registrations and grants or other funding). Any other potential conflict of interest is disclosed.

\section{References}

1. Dhawan AK, Verma P, Sharma S. Oral lesions in leprosy revisited: a case report. Am J Dermatopathol. 2012;34:666-7. doi:10.1097/ DAD.0b013e3182485bcc.

2. Ali K, Sittampalam G, Malik MA. Facial tuberculoid leprosy: case report. Br J Oral Maxillofac Surg. 2011;49:70-2. doi:10.1016/j. bjoms.2010.01.011.

3. Scollard DM, Skinsnes OK. Oropharyngeal leprosy in art, history, and medicine. Oral Surg Oral Med Oral Pathol Oral Radiol Endod. 1999;87:463-70.

4. Böddinghaus BK, Ludwig RJ, Kaufmann R, Enzensberger R, Gies $\mathrm{V}$, Kramme S, Brade V, Brandt CM. Leprosy in a pregnant woman. Infection. 2007;35:37-9. 

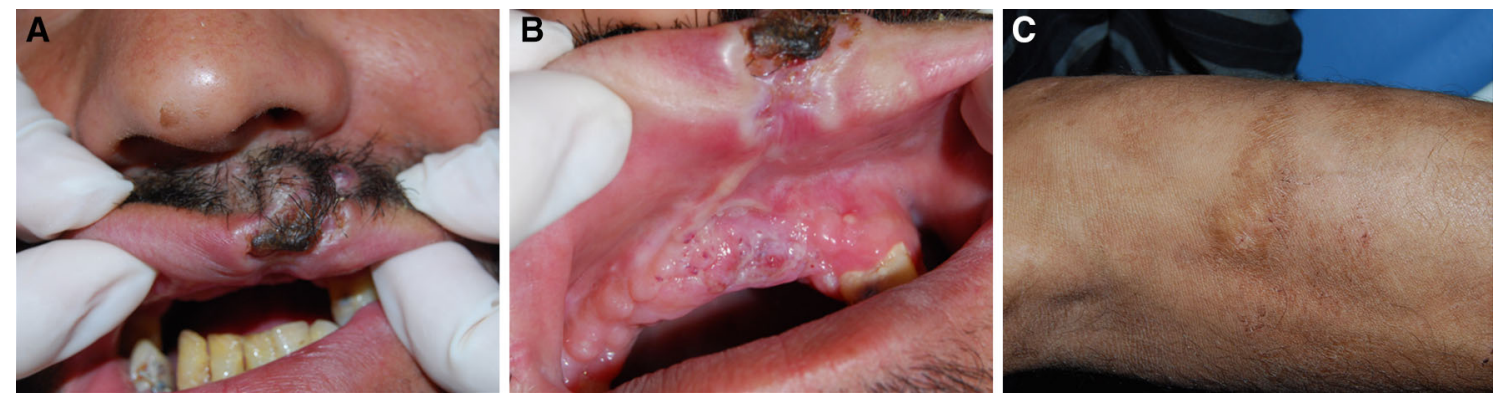

Fig. 1 a Painless dry ulcer on the upper lip covered with black necrotic material. b An asymptomatic, pink granulomatous lesions involving anterior alveolar gingiva. c Hypopigmented patched lesions in the skin of the upper limb

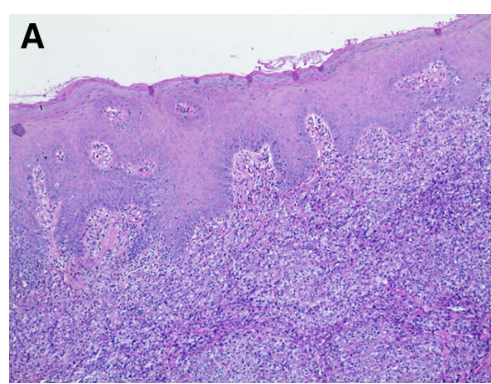

Fig. 2 a Histological section stained with $H \& E(\times 40)$ showed subepithelial inflammatory infiltrate. b Dense infiltrate of prominent vacuolated-foam macrophage, occasional lymphocytes and rare
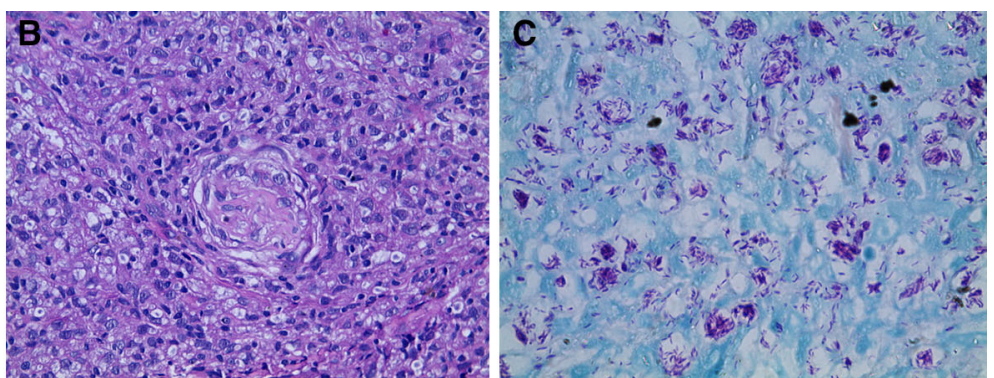

Langhans-type giant cells could be seen without the typical granuloma arrangement $(\mathrm{H} \& \mathrm{E}, 400 \times)$. c Ziehl-Neelsen staining $(\times 1000)$ showed numerous acid-fast bacilli arranged as globi 